\title{
ESTABELECIMENTO E REORGANIZAÇÃO DE RELAÇÕES ARBITRÁRIAS DERIVADAS APÓS O TREINO EM MATCHING-TO-SAMPLE DE IDENTIDADE COM CONSEQUÊNCIAS ESPECÍFICAS
}

\section{ESTABLISHMENT AND REORGANIZATION OF DERIVED ARBITRARY RELATIONS FOLLOWING IDENTITY MATCHING-TO-SAMPLE TRAINING WITH SPECIFIC CONSEQUENCES}

\author{
MARCELO V. SILVEIRA' \\ HARRY A. MACKAY \\ JULIO C. DE ROSE ${ }^{1}$

\section{(1) Universidade Federal de SÃo CaRlos e Instituto Nacional de CIÊNCIA E TECNOlogia Sobre Comportamento, COGNIÇÃO E ENSINO, BRASIL}

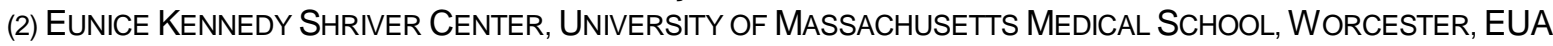

\section{RESUMO}

No Experimento 1, seis participantes foram treinados em um procedimento MTS de identidade (IDMTS) para estabelecer relações estímulo-estímulo A1A1, A2A2, A3A3; B1B1, B2B2, B3B3; e C1C1, C2C2, C3C3. Respostas corretas resultavam na apresentação de consequências específicas, $\mathrm{Sr} 1, \mathrm{Sr} 2$ e Sr3. Uma resposta de consumação às consequências específicas foi requerida para que os participantes pudessem coletar os pontos nas tentativas de treino. O procedimento MTS arbitrário foi utilizado para documentar a formação de três classes de equivalência A1B1C1Sr1, A2B2C2Sr2 e A3B3C3Sr3. Em uma fase subsequente, o IDMTS com consequências específicas foi empregado para treinar as relações D1D1, D2D2 e D3D3. Os estímulos D eram fotografias de faces humanas expressando alegria (D1), tristeza (D2) e neutralidade (D3). Por último, avaliações por meio do diferencial semântico atestaram a ocorrência da transferência das funções (i.e., "significados") dos estímulos D para os estímulos C e para $\mathrm{Sr} 1$ e Sr3. Estes resultados sugeriram que as classes ABCSr se expandiram para incluir os estímulos significativos D (i.e., A1B1C1D1Sr1, A2B2C2D2Sr2 e A3B3C3D3Sr3). No Experimento 2 verificou-se que, para cinco participantes, o procedimento de reversão das consequências específicas $\mathrm{Sr} 1$ e Sr2 em relação aos estímulos C1, C2 e D1 e D2 deu origem a duas classes de equivalência reorganizadas (A1B1C2D2Sr2 e A2B2C1D1Sr1). Paralelamente, os dados do diferencial semântico documentaram a reversão dos significados atribuídos previamente aos estímulos D1 e D2. Estes achados sugerem que o procedimento de reversão reorganizou as classes de estímulos bem como seus significados. Porém, resultados análogos não foram reportados para as consequências específicas.

Palavras-chave: Matching-to-sample de identidade, consequências específicas, classes de equivalência, reorganização de classes de equivalência, transferência de função, universitários

In Experiment 1, six participants were given identity matching to sample (IDMTS) training that established the stimulusstimulus relations $\mathrm{A} 1 \mathrm{~A} 1, \mathrm{~A} 2 \mathrm{~A} 2, \mathrm{~A} 3 \mathrm{~A} 3$; $\mathrm{B} 1 \mathrm{~B} 1, \mathrm{~B} 2 \mathrm{~B} 2, \mathrm{~B} 3 \mathrm{~B} 3$; and $\mathrm{C} 1 \mathrm{C} 1, \mathrm{C} 2 \mathrm{C} 2, \mathrm{C} 3 \mathrm{C} 3$. Correct responses resulted in presentations of class-specific consequences, $\mathrm{Sr} 1, \mathrm{Sr} 2$, and $\mathrm{Sr} 3$. Consummatory responses to the class-specific consequences were required to collect points on training trials. Subsequent tests used arbitrary matching to sample to confirm the formation of three equivalence classes, $\mathrm{A} 1 \mathrm{~B} 1 \mathrm{C} 1 \mathrm{Sr} 1, \mathrm{~A} 2 \mathrm{~B} 2 \mathrm{C} 2 \mathrm{Sr} 2$, and $\mathrm{A} 3 \mathrm{~B} 3 \mathrm{C} 3 \mathrm{Sr} 3$. Next, IDMTS, with the specific consequences, was used to train D1D1, D2D2 and D3D3 relations. These D stimuli were pictures of human faces portraying happiness (D1), sadness (D2), and neutrality (D3). Finally, tests used semantic differential ratings to document transfer of functions (i.e., "meanings") of the D stimuli to the $\mathrm{C}$ stimuli and to the $\mathrm{Sr} 1$ and $\mathrm{Sr} 3$ stimuli, in accord with class membership. These results suggest expansion of the $\mathrm{ABCSr}$ classes to include the meaningful D stimuli (i.e., A1B1C1D1Sr1, A2B2C2D2Sr2, and A3B3C3D3Sr3). Experiment 2 verified that, for five participants, reversals of the class-specific consequences $\mathrm{Sr} 1$ and $\mathrm{Sr} 2$ with respect to stimuli $\mathrm{C} 1$, and $\mathrm{C} 2$, and D1 and D2, gave rise to the two reorganized equivalence classes A1B1C2D2Sr2 and A2B2C1D1Sr1. Semantic differential ratings then documented reversals of the meanings given to stimuli $\mathrm{C} 1$ and $\mathrm{C} 2$. Also, semantic differential ratings documented the reversals of the meanings of $\mathrm{C}$ stimuli in accordance with their reorganized class-memberships with D stimuli. However, analogous reversals of function transfer were not observed for the class-specific consequences.

Key words: Identity matching-to-sample, class-specific consequences, equivalence classes, reorganization of equivalence classes, transfer of functions, undergraduates.

\footnotetext{
Parte dos resultados apresentados nesse manuscrito foram obtidos da tese de doutorado do primeiro autor. Aproveitamos esta oportunidade para agradecer aos membros de sua banca Lidia Postalli, Raquel Melo, Renato Bortoloti, João de Almeida, e estender os agradecimentos a Bill Dube, Bill Mcllvane, Brooks Thompson. A pesquisa é parte do programa científico do Instituto Nacional de Ciência e Tecnologia sobre Comportamento Cognição e Ensino (INCT-ECCE), apoiado pelo Conselho Nacional de Desenvolvimento Científico e Tecnológico (CNPq: Processo \#573972/2008-7) e pela Fundação de Amparo à Pesquisa do Estado de São Paulo (FAPESP: Processo \#2008/57705-8). Agradecemos à Deisy das Graças de Souza, coordenadora do INCT-ECCE, por ter gentilmente cedido o software Gerenciador Individualizado de Ensino por Computador (FAPESP: Processo \#2005/60652-5), pelo incentivo à condução desta pesquisa e por encorajar a publicação do material produzido. O primeiro autor agradece à FAPESP pelos auxílios (Bolsa de Doutorado \#2011/12847-2, Bolsa de Estágio em Pesquisa no Exterior \#2015/08322-8). O segundo autor agradece ao Kennedy Shriver Center, New England Center for Children. O terceiro autor é Bolsista Produtividade pelo CNPq.
} 
Relações derivadas reflexivas, simétricas e transitivas podem decorrer do ensino de relações condicionais por meio do procedimento matching-tosample (MTS) arbitrário. Este procedimento pode ser programado de modo que a apresentação de uma consequência (e.g., pontos, moedas ou tokens) seja contingente à emissão do padrão de respostas aos estímulos experimentalmente definidos como "corretos". Por exemplo, diante do estímulo modelo A1, escolher os estímulos de comparação B1 e C1, não B2 e C2; e diante do estímulo modelo A2, escolher os estímulos de comparação B2 e C2, não B1 e C1. As escolhas de B2 e $\mathrm{C} 2$ diante do modelo $\mathrm{A} 1$ e de $\mathrm{B} 1$ e $\mathrm{C} 1$ diante do modelo A2 (i.e., padrão de respostas aos estímulos experimentalmente definidos como "incorretos") resultam na apresentação de outro tipo de consequência (e.g., timeout). Após o ensino direito das relações condicionais A1B1, A2B2, A1C1 e A2C2, observa-se que, sem a necessidade de treino adicional, o comportamento passa a ser controlado por relações reflexivas (A1A1, A2A2, B1B1, B2B2, C1C1 e C2C2), relações simétricas (B1A1, B2A2, C1A1 e C2A2) e relações transitivas (B1C1, B2C2, C1B1 e C2B2). A emergência de relações reflexivas, simétricas e transitivas atestam a formação de classes de equivalência (Sidman \& Tailby, 1982). É importante observar que no contexto do ensino de relações arbitrárias estas relações derivadas são esperadas porque os elementos mais salientes das contingências que sinalizam o reforçamento diferencial encontram-se nas relações entre os estímulos antecedentes As, Bs e Cs.

Uma maneira alternativa de se estabelecer comportamentos controlados por relações derivadas consiste em submeter os sujeitos experimentais a contingências nas quais as relações entre os estímulos antecedentes e as consequências diferenciais sejam salientes (cf., Barros, Lionello-DeNolf, Dube, \& McIlvane, 2006; Dube, McIlvane, Mackay, \& Stoddard, 1987; Dube, McIlvane, Maguire, Mackay, \& Stoddard, 1989; Goyos, 2000; Johnson, Meleshkevich, \& Dube, 2014; Joseph, Overmeier, \& Thompson, 1998; Minster, Jones, Ellife, \& Muthukumaraswamy, 2006; Monteiro \& Barros, 2016; Schenk, 1994; Varella \& de Souza, 2014, 2015). No Experimento 2 do estudo de Schenk (1994), por exemplo, oito crianças foram submetidas ao procedimento MTS de identidade no qual a apresentação de dois tipos de consequências (i.e., bolinhas azuis e vermelhas) era contingente à escolha dos estímulos de comparação idênticos ao estímulo modelo. Uma bolinha azul (Sr1) era entregue sempre que as comparações A1, B1, C1 e D1 eram escolhidas e uma bolinha vermelha (Sr2) era entregue sempre que as comparações A2, B2, C2 e D2 eram escolhidas. Após aprenderem as relações de identidade, Schenk (1994) observou que o responder dos sujeitos passou a ser controlado por relações arbitrárias B1A1, B2A2, C1A1, C2A2, D1A1, D2A2, B1C1, B2C2, C1B1, C2B2, B1D1, B2D2, D1B1, D2B2, C1D1, C2D2, D1C1 e D2C2. Desempenhos análogos aos do Experimento 2 de Schenk (1994) foram obtidos por Goyos (2000) com crianças pré-escolares, e por Dube e
McIlvane (1995), Varella e de Souza (2014, 2015) e Monteiro e Barros (2016) com indivíduos com desenvolvimento atípico. Estas relações arbitrárias emergentes a partir de um treino de MTS de identidade, com consequências específicas, têm sido categorizadas como relações transitivas. Em tais casos, as consequências específicas atuam como elementos comuns (nódulos) para a emergência de novas relações entre estímulos antecedentes que nunca haviam sido diretamente relacionados (cf. Dube et al., 1987, 1989; Sidman, 1994, 2000).

Porém, em um estudo mais recente, Silveira e de Rose (2015) observaram que humanos adultos fracassaram sistematicamente em exibirem a emergência de relações arbitrárias após o treino em MTS de identidade com consequências específicas. No Experimento 1, cinco universitários foram submetidos a um procedimento MTS de identidade envolvendo um conjunto de estímulos abstratos (B1, B2, B3, C1, C2 e C3) e figuras de faces humanas expressando alegria (A1), neutralidade (A2) e raiva (A3). Na primeira fase do experimento, os participantes foram treinados a responder ao estímulo de comparação idêntico ao estímulo modelo. Quando o responder dos participantes satisfazia esta condição, uma animação e uma melodia eram produzidas pelo computador por $1,5 \mathrm{~s}$. Respostas aos estímulos da Classe 1 (i.e., A1, B1 e C1) produziam estrelas em movimento e um tipo de melodia ( $\mathrm{Sr} 1)$. Quadrados em preto e branco, simulando o movimento em um corredor, juntamente com outra melodia e um tabuleiro de xadrez juntamente com outra melodia ( $\mathrm{Sr} 2$ e $\mathrm{Sr} 3$, respectivamente) eram apresentados quando os participantes respondiam aos estímulos das Classes 2 e 3 , respectivamente. Após o treino, os participantes foram submetidos a tentativas que testaram a emergência de relações arbitrárias $\mathrm{AB}, \mathrm{BA}, \mathrm{AC}, \mathrm{CA}, \mathrm{BC}$ e CB. Porém, as referidas relações não emergiram para nenhum dos participantes. No Experimento 2 do referido estudo, outros dois participantes exibiram baixos escores de acertos nas tentativas que testavam a emergência das mesmas relações, mesmo após terem sido expostos a um super-treino das relações de identidade com as consequências específicas.

É possível que as consequências ( $\mathrm{Sr} 1, \mathrm{Sr} 2$ e Sr3) utilizadas por Silveira e de Rose (2015) não tenham atuado com consequências específicas para respostas de escolha experimentalmente definidas como corretas. Isso pode ter ocorrido porque, diferentemente de outros estudos na área (e.g., Dube et al., 1989; Goyos, 2000; Johnson et al., 2014; Schenk, 1994), as Sr1, Sr2 e Sr3 selecionados por Silveira e de Rose (2015) não tinham qualquer relação com reforçadores secundários e primários para indivíduos desta população. Assim, todas as Srs poderiam estar sinalizando o encerramento de uma tentativa, dado que esta seria uma consequência comum para todas as tentativas de MTS de identidade nas quais os participantes emitiam as respostas experimentalmente definidas como corretas. Deste modo, ainda que os participantes tivessem atingido o critério de aprendizagem no treino, a ineficácia dessas Srs para 
atuarem como consequências específicas no contexto do treino em MTS de identidade estabeleceria ocasião para que os participantes respondessem indiscriminadamente nas tentativas de teste em MTS arbitrário.

$\mathrm{Na}$ presente pesquisa os estímulos empregados como consequências específicas por Silveira e de Rose (2015) foram substituídos por estímulos correlacionados com o acesso a reforçadores primários (lanches e bebidas) e secundários (e.g., material escolar e livros). Além disso, durante o treino, foi acrescida uma exigência para que os participantes respondessem aos estímulos selecionados para serem usados como consequências. Essa exigência aumentou a probabilidade de emissão de respostas de observação às propriedades visuais desses estímulos. E, mais do que isso, seria uma maneira de garantir que os participantes aprendessem a responder diferencialmente às relações entre os estímulos antecedentes e os estímulos que atuavam como consequências específicas. O objetivo foi verificar se, dadas estas manipulações, os participantes treinados em procedimento MTS de identidade com consequências específicas exibiriam um responder controlado por relações arbitrárias em tentativas de teste.

\section{EXPERIMENTO 1 MÉTODO}

\section{Participantes}

Participaram do experimento 16 estudantes universitários, experimentalmente ingênuos, com idades entre 18 e 22 anos. Os procedimentos aos quais os participantes foram submetidos, bem como os termos expressos no TCLE, e a viabilidade da condução da pesquisa no referido local, foram avaliadas e devidamente aprovadas pela Comissão de Ética em Pesquisa com Seres Humanos da UFSCar (CAAE \#01830112.6.0000.5504). A duração do Experimento era de 80 minutos. Os participantes do Grupo Experimental $(\mathrm{N}=6)$ foram submetidos aos procedimentos de MTS. Os participantes que integraram o Grupo Controle $(\mathrm{N}=10)$ foram convidados a permanecerem em sala de aula por aproximadamente 10 minutos após o término de uma aula.

\section{Materiais, Estímulos e Local}

O procedimento com o Grupo Experimental era realizado em um cubículo do Laboratório de Estudos do Comportamento Humano da UFSCar (LECH-UFSCar). Um computador Apple Macintosh MAC OS foi utilizado. O software Gerenciador de Ensino Individualizado por Computadorizado - GEIC desenvolvido por Capobianco et al. (2009), foi utilizado para a apresentação dos estímulos e registro das respostas dos participantes. Seis desses estímulos eram figuras abstratas desenhadas na cor preta sobre um fundo branco (A1, A2, A3, B1, B2, $\mathrm{B} 3, \mathrm{C} 1, \mathrm{C} 2$ e C3), obtidas do arquivo de imagens do $M A C$ $O S$ (ver Figura 1). Os outros três estímulos (D1, D2 e D3) eram faces humanas expressando alegria, tristeza e raiva, obtidas do Pictures of Facial Affect $@$ CD-ROM (www.paulekman.com).

Consequências específicas. As consequências específicas eram formadas por estímulos auditivos (EAs) distintos (i.e., EA 1, EA 2 e EA 3) combinados a três logotipos diferentes de lojas situadas na Universidade. Os logotipos representavam uma loja de material escolar (Fast Copy - Sr1), uma lanchonete (PQ - Sr2), e uma loja de livros (EDUFSCar - Sr3).

\section{Procedimentos pré-experimentais}

Instruções escritas. Antes de serem conduzidos ao cubículo, os participantes do Grupo Experimental recebiam o TCLE. Nesse documento os participantes encontravam informações gerais sobre a tarefa e sobre o fato de que durante a execução do procedimento eles obteriam pontos por responder de modo correto. O documento especificava ainda que cada ponto poderia valer $\mathrm{R} \$ 0,05, \mathrm{R} \$ 0,07$ e $\mathrm{R} \$ 0,10$ e que esses valores acumulados poderiam ser trocados por vales-brinde ao final do experimento. Após a leitura do TCLE, o experimentador entregava uma outra folha aos participantes para que pudessem especificar os valores dos pontos para cada uma das $\mathrm{Srs}^{1}$. O experimentador permanecia na sala junto com os participantes ao longo de toda a fase pré-experimental, para sanar eventuais dúvidas.

Atribuição de valor aos pontos. Após a leitura das instruções escritas, o experimentador entregava mais uma folha aos participantes (cf. Almeida, Bortoloti, Ferreira, Schelini, \& de Rose, 2014). Esta folha continha instruções adicionais sobre os valores que cada ponto poderia valer $(\mathrm{R} \$ 0,05 ; \mathrm{R} \$ 0,07$ e $\mathrm{R} \$ 0,10)$. Os participantes foram informados que, ao final do experimento, eles teriam acesso a um relatório indicando os valores em dinheiro que haviam obtido para cada um dos logotipos. Ao final do experimento, o valor acumulado para a loja preferida dos participantes era escrito em um vale-brinde.

\section{Procedimentos experimentais}

Características Gerais do Procedimento MTS. O procedimento MTS foi utilizado nas fases de treino e teste. As tentativas se iniciavam com a apresentação do estímulo-modelo na porção central da tela. Uma resposta de observação sobre o modelo resultava na sua remoção e, em seguida três estímulos de comparação eram apresentados na porção inferior da tela. Ao longo das fases de treino e teste, os participantes utilizaram um mouse sem fio para responder aos estímulos modelos e aos estímulos de comparação. Durante o treino de linha de base as respostas aos estímulos de comparação "corretos" eram seguidas pelas consequências específicas. Não haviam consequências programadas para as respostas aos estímulos de comparação "incorretos".

\footnotetext{
${ }^{1}$ Para ter acesso a estas instruções, entre em contato com o primeiro autor.
} 


\section{Classe 1}

Classe 2

Classe 3

A
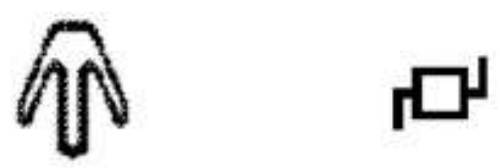

$J \cdot C$

B

un

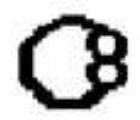

$\not 8$

C
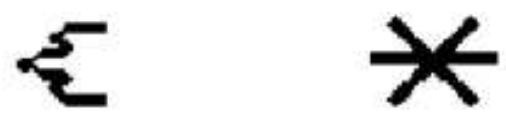

G

D
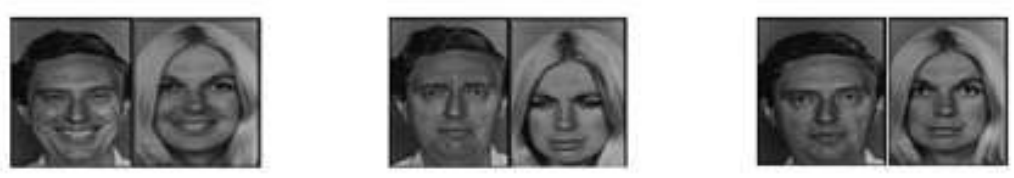

\section{Consequências Especificas}
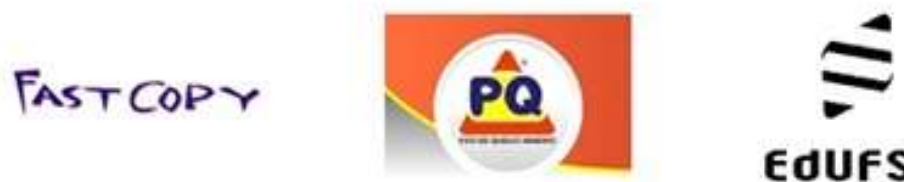

EdUfSCar

Figura 1. Estímulos antecedentes (A, B, C e D) e logotipos que formavam as consequências específicas (Srs) em ambos os estudos.

Consequências específicas. Quando o estímulo de comparação "correto" era selecionado, um EA com duração de 1,5 segundos era imediatamente apresentado. A apresentação de EA1 era contingente à emissão de respostas corretas aos estímulos da Classe 1 (A1, B1, C1 e D1). O EA2 e o EA3, eram contingentes à emissão de respostas corretas aos estímulos da Classe 2 (A2, B2, C2 e D2) e Classe 3 (A3, B3, C3 e D3), respectivamente. Após a apresentação de um EA, um dos logotipos previamente selecionados para atuarem como consequências específicas - FastCopy (Sr1), PQ (Sr2), ou EDUFSCar (Sr3) - era apresentado na posição inferior da tela do computador. Um contador para os pontos obtidos estava localizado acima do logotipo. O logotipo permanecia no monitor até o momento em que o participante posicionasse o cursor do mouse sobre ele e clicasse. Essa resposta de consumação era requerida para que os participantes pudessem acumular pontos (cf. Costa, Patsko, \& Becker, 2007). Sempre que a resposta de consumação era emitida, um ponto era acrescentado no contador e o mesmo EA era apresentado novamente. Em seguida, uma nova tentativa se iniciava. As respostas "incorretas" não acarretavam na perda de pontos previamente ganhos. 


\section{Treino}

Linha de Base. O procedimento MTS de identidade com consequências específicas foi empregado para estabelecer as relações de identidade AA, BB, CC e DD. Cada bloco de treino tinha 12 tentativas e, para avançar de um bloco para outro os participantes deveriam acertar, no mínimo, 92\% das tentativas (11 acertos) em cada bloco. Ao final de um bloco de treino a sessão era momentaneamente interrompida para que o experimentador verificasse se o participante atingiu o critério de aprendizagem.

Linha de Base Cumulativa. Os blocos de Linha de Base Cumulativa eram compostos por tentativas das relações previamente treinadas e eram apresentados antes das tentativas de teste. Nos blocos de Linha de Base Cumulativa, as Srs continuavam a ser apresentadas mediante respostas de escolha ao estímulo de comparação idêntico ao estímulo modelo. Desta maneira, buscou-se aumentar a probabilidade de formação de classes de equivalência entre os participantes do Grupo Experimental (cf. Arntzen, 2012).

A Linha de Base Cumulativa 1 integrou 12 tentativas AA e 12 tentativas $\mathrm{BB}$, totalizando 24 tentativas. A Linha de Base Cumulativa 2 era composta por 36 tentativas: 12 AA, 12 BB e 12 CC. A Linha de Base Cumulativa 3 constituiu a última fase de treino e foi conduzida somente após a realização de todos os testes. O bloco era composto por 48 tentativas: $12 \mathrm{AA}, 12 \mathrm{BB}, 12$ $\mathrm{CC}$ e 12 DD. Para encerrar qualquer um dos blocos de linha de base cumulativa os participantes deveriam acertar $100 \%$ das tentativas. Os participantes eram submetidos aos blocos de testes tão logo o critério nos blocos de Linha de Base Cumulativa fosse atingido.

\section{Testes}

Testes de emergência de relações arbitrárias. As tentativas de teste eram apresentadas em blocos de 15 tentativas. Após a Linha de Base Cumulativa 1, os participantes eram submetidos aos testes AB (A1B1, A2B2 e A3B3) e BA (B1A1, B2A2 e B3C3). Após a Linha de Base Cumulativa 2, os participantes eram submetidos aos blocos de teste AC (A1C1, $\mathrm{A} 2 \mathrm{C} 2$ e $\mathrm{A} 3 \mathrm{C} 3), \mathrm{CA}$ (C1A1, C2A2 e C3A3), BC (B1C1, B2C2 e B3C3), e CB (C1B1, C2B2 e C3B3). Três blocos adicionais $\mathrm{SrA}, \mathrm{SrB}$ e $\mathrm{SrC}$ verificaram a emergência das relações Sr1A1, Sr2A2 e Sr3A3; Sr1B1, Sr2B2 e Sr3B3; e Sr1C1, $\mathrm{Sr} 2 \mathrm{C} 2$ e Sr3C3. O critério para determinar se os desempenhos nos testes denotavam a emergência de relações arbitrárias era a obtenção de, pelo menos, $90 \%$ de respostas consistentes com a linha de base (13 "acertos) em todos os blocos
(Arntzen, 2012). Os estímulos Ds não foram apresentados nos blocos de teste para evitar que os estímulos abstratos assumissem as propriedades afetivas das faces por meio de exposição direta, estabelecida no contexto das tentativas de MTS.

Diferencial Semântico. Cópias do instrumento validado por Almeida et al. (2014) foram entregues aos participantes do Grupo Experimental e do Grupo Controle. O estímulo a ser avaliado ficava na parte superior da folha e as 13 escalas ficaram logo abaixo dele, ocupando toda a posição central da folha. Cada uma das escalas compreendia sete valores $(-3 ;-2 ;-1 ; 0 ; 1$; $2 ; 3)$. Nas extremidades de cada escala (-1 e 3$)$ estavam ancorados um adjetivo e o seu antônimo (e.g., feio-bonito; agradável-desagradável)..

\section{Aplicação do Diferencial Semântico}

Grupo Experimental. Os participantes que atingiram os critérios nas fases de MTS foram conduzidos a uma sala separada na qual puderam avaliar os estímulos C1, C2, C3, Sr1, $\mathrm{Sr} 2$ e Sr3 por meio do diferencial semântico. O diferencial semântico continha uma folha com instruções ${ }^{2}$ que deveriam ser lidas antes do início do preenchimento das escalas.

Grupo Controle. Os participantes deste grupo avaliaram os estímulos Ds utilizando o diferencial semântico. Esses participantes estavam dentro de sua própria sala de aula e foram instruídos a preencherem as escalas individualmente, após terem lido a folha com instruções.

\section{RESULTADOS \\ Estabelecimento de relações arbitrárias a partir do procedimento MTS de identidade com consequências específicas}

A Tabela 1 mostra as porcentagens de acertos obtidas pelos participantes nos blocos de treino, linhas de base cumulativa e testes. Todos os participantes atingiram o critério no Treino AA, Treino BB e Linha de Base Cumulativa 1. Porém, apenas P6 obteve altos escores de acertos nos blocos de teste AB, BA, SrA e SrB. Os participantes P1, P2, P3, P4 e P5 foram submetidos mais uma vez ao bloco de Linha de Base Cumulativa $1 \mathrm{e}$, em seguida aos blocos de testes. As porcentagens de acertos neste retreino estão indicadas na Tabela 1 pela nota ${ }^{a}$. Todos os participantes atingiram os critérios para a Linha de Base Cumulativa 1 e também para os blocos de AB, BA, SrA e SrB. Em seguida, os participantes foram submetidos a um treino envolvendo novos estímulos abstratos (D1, D2 e D3). Os participantes atingiram $100 \%$ de acertos

\footnotetext{
2 Para acesso às instruções, por favor, entre em contato com o primeiro autor.
} 
no Treino $\mathrm{CC}$, na Linha de Base Cumulativa 2 e nos testes AC, CA, BC, CB e SrC. Por último, os participantes foram submetidos ao Treino DD (com figuras de faces humanas expressando emoções) e à Linha de Base Cumulativa 3. Todos obtiveram $100 \%$ de acerto nestes blocos.

Tabela 1.

Porcentagens de Acertos dos Participantes nos Blocos de Treino e Teste do Experimento 1.

\begin{tabular}{|c|c|c|c|c|c|c|c|}
\hline \multirow[b]{2}{*}{ Fases } & \multirow[b]{2}{*}{ Critério } & \multicolumn{6}{|c|}{ Participantes } \\
\hline & & P1 & P2 & P3 & $\mathrm{P} 4$ & P5 & P6 \\
\hline Treino AA & 92 & 100 & 100 & 100 & 100 & 100 & 100 \\
\hline Treino BB & 92 & 96 & 100 & 100 & 100 & 100 & 100 \\
\hline Linha de Base & \multirow{2}{*}{100} & 100 & \multirow{2}{*}{100} & 97 & 100 & 100 & \multirow{2}{*}{100} \\
\hline Cumulativa 1 & & $100^{\mathrm{a}}$ & & $100^{\mathrm{a}}$ & $100^{a}$ & $100^{\mathrm{a}}$ & \\
\hline \multirow{2}{*}{ Teste AB } & \multirow{2}{*}{90} & 66 & 97 & 50 & 33 & 33 & \multirow{2}{*}{100} \\
\hline & & $100^{\mathrm{a}}$ & $100^{\mathrm{a}}$ & $100^{\mathrm{a}}$ & $100^{\mathrm{a}}$ & $100^{\mathrm{a}}$ & \\
\hline \multirow{2}{*}{ Teste BA } & \multirow{2}{*}{90} & 41 & 17 & 33 & 100 & 42 & \multirow{2}{*}{100} \\
\hline & & $100^{\mathrm{a}}$ & $100^{\mathrm{a}}$ & $100^{\mathrm{a}}$ & $100^{\mathrm{a}}$ & $100^{\mathrm{a}}$ & \\
\hline \multirow{2}{*}{ Teste SrA } & \multirow{2}{*}{90} & 33 & 0 & 16 & 100 & 100 & \multirow{2}{*}{100} \\
\hline & & $100^{\mathrm{a}}$ & $100^{\mathrm{a}}$ & $100^{\mathrm{a}}$ & $100^{\mathrm{s}}$ & $92^{\mathrm{a}}$ & \\
\hline \multirow{2}{*}{ Teste SrB } & \multirow{2}{*}{90} & 100 & 33 & 9 & \multirow{2}{*}{100} & 33 & \multirow{2}{*}{100} \\
\hline & & $100^{\mathrm{a}}$ & $100^{\mathrm{a}}$ & $100^{\mathrm{a}}$ & & $100^{\mathrm{a}}$ & \\
\hline \multirow{2}{*}{ Treino CC } & \multirow{2}{*}{92} & \multirow{2}{*}{100} & 33 & \multirow{2}{*}{100} & \multirow{2}{*}{100} & \multirow{2}{*}{100} & \multirow{2}{*}{100} \\
\hline & & & $100^{\mathrm{a}}$ & & & & \\
\hline Linha de Base & \multirow{2}{*}{100} & \multirow{2}{*}{100} & \multirow{2}{*}{100} & \multirow{2}{*}{100} & \multirow{2}{*}{100} & \multirow{2}{*}{100} & \multirow{2}{*}{100} \\
\hline Cumulativa 2 & & & & & & & \\
\hline Teste AC & 90 & 100 & 100 & 100 & 100 & 100 & 100 \\
\hline Teste CA & 90 & 100 & 100 & 100 & 100 & 100 & 100 \\
\hline Teste BC & 90 & 100 & 100 & 100 & 100 & 100 & 100 \\
\hline Teste CB & 90 & 100 & 100 & 100 & 100 & 100 & 100 \\
\hline Teste $\mathrm{SrC}$ & 90 & 100 & 100 & 100 & 100 & 100 & 100 \\
\hline Treino DD & 92 & 100 & 100 & 100 & 100 & 100 & 100 \\
\hline $\begin{array}{l}\text { Linha de Base } \\
\text { Cumulativa } 4\end{array}$ & 100 & 100 & 100 & 100 & 100 & 100 & 100 \\
\hline
\end{tabular}

${ }^{\text {a }}$ Retreino e reteste.

\section{Análise dos diferenciais semânticos}

A Figura 2 mostra as medianas das avaliações feitas dos estímulos C1, C2 e C3 pelos participantes do Grupo Experimental (linha tracejada) e as medianas das avaliações feitas pelo Grupo Controle dos estímulos D1, D2 e D3 (linha cheia). Para facilitar a apresentação dos resultados, os estímulos C1 e D1 serão chamados de "Classe Alegre", os estímulos C2 e D2 serão chamados de "Classe Triste" e os estímulos C3 e D3 serão chamados de "Classe Neutra". Além disso, as escalas foram reorganizadas de modo que os adjetivos negativos ficassem todos situados do lado esquerdo e os estímulos positivos ficassem do lado direito. As escalas também foram dividias em fatores: Fator 1 e Fator 2, sendo o primeiro chamado "Avaliação" e o segundo, "Potência". Na presente pesquisa, as escalas do Fator 1 serão analisadas. De acordo com Almeida et al. (2014) as escalas do Fator 1 permitem aferir a valência afetiva dos estímulos (Coeficiente alpha de Cronbach: 0,91), que é uma das variáveis dependentes relevantes desse estudo. De modo contrário, foi verificado que as 
escalas do Fator 2 são sensíveis às dimensões potência e atividade (Coeficiente alpha de Cronbach: 0,62). Por esse motivo, as escalas do Fator 2 foram consideradas como distratoras.

As escalas à esquerda da Figura 2 mostram as avaliações feitas dos estímulos da Classe Alegre. As medianas das avaliações do estímulo C1 pelo Grupo Experimental em cada uma das nove escalas do Fator 1 foram as seguintes: "triste-alegre": +1; "tenso-relaxado": +1; “áspero-liso": +2; "feio-bonito": $+1,5$; "pesado-leve": +1,5; "negativo-positivo": +2; "duro-macio": $\quad+0,5$; "mau-bom": $\quad+2$; "desagradável-agradável": +2,5. Os valores das medianas das avaliações dos estímulos D1 feitas pelo Grupo Controle foram os seguintes: "tristealegre": +1,5; "tenso-relaxado": +2; "áspero-liso": 0; "feio-bonito": 0; "pesado-leve": 0; "negativopositivo": +2; "duro-macio": 0; "mau-bom": +1; "desagradável-agradável": +1,5. Portanto, as medianas dos valores das avaliações dos estímulos C1 e D1 feitas pelos dois grupos situaram-se entre 0 e +3 , havendo sobreposição de valores apenas na escala "positivo-negativo". Estes resultados mostram que ambos os estímulos possuíam valências afetivas positivas.

As escalas ao centro mostram as avaliações dos estímulos da Classe Triste. As medianas das avaliações do estímulo C2 pelo Grupo Experimental em cada uma das nove escalas do Fator 1 foram os seguintes: "tristealegre": -2; "tenso-relaxado": -2,5; "áspero-liso": -0.5; "feio-bonito": -0,5; "pesado-leve": -1; "negativo-positivo": 0; "duro-macio": -1,5; "maubom": -1; “desagradável-agradável": -0,5. Os valores das medianas das avaliações dos estímulos D2 feitas pelo Grupo Controle foram os seguintes: "triste-alegre": -3; "tenso-relaxado": -3; “ásperoliso": -2; "feio-bonito": -2; "pesado-leve": -2; "negativo-positivo": -2,5; "duro-macio": 0; "maubom": -2; "desagradável-agradável": -2. Portanto, as medianas dos valores das avaliações dos estímulos C2 e D2 feitas pelos dois grupos situaram-se entre 0 e -3 . Não houve sobreposição de valores em nenhuma das escalas do Fator 1. Os resultados mostram que ambos os estímulos possuíam valências afetivas negativas.

As escalas à direita mostram as avaliações dos estímulos da Classe Neutra. As medianas das avaliações do estímulo C3 pelo Grupo Experimental em cada uma das nove escalas do Fator 1 foram as seguintes: "triste-alegre": +1; "tenso-relaxado": 0; "áspero-liso": +0.5; "feiobonito": +1; "pesado-leve": +1; "negativopositivo": +1,5; "duro-macio": 0; "mau-bom": 0; "desagradável-agradável": +1. Os valores das medianas das avaliações dos estímulos D3 feitas pelo Grupo Controle foram os seguintes: "tristealegre": 0; "tenso-relaxado": -0,5; "áspero-liso": 0; "feio-bonito": 0; "pesado-leve": 0; "negativo- positivo": -1; "duro-macio": 0; "mau-bom": -1; "desagradável-agradável": $\quad-1$. Portanto, as medianas dos valores das avaliações dos estímulos C3 e D3 feitas pelos dois grupos situaram-se entre -1 e $+1,5$. Houve sobreposição de valores apenas na escala "duro-macio". Estes resultados mostram que ambos os estímulos possuíam valências neutras.

A Figura 3 mostra os valores das medianas das avaliações dos estímulos $\mathrm{Sr} 1, \mathrm{Sr} 2$ e $\mathrm{Sr} 3$, pelo Grupo Experimental e as medianas das avaliações dos estímulos D1, D2 e D3 pelo Grupo Controle. De modo análogo, as Srs serão designadas conforme a sua relação com cada classe de estímulos: Classe Alegre ( $\mathrm{Sr} 1)$, Classe Triste (Sr2) e Classe Neutra (Sr3).

As escalas à esquerda da Figura 3 mostram as avaliações dos estímulos da Classe Alegre. As medianas das avaliações do estímulo Sr1 pelo Grupo Experimental em cada uma das nove escalas do Fator 1 foram as seguintes: "tristealegre": +2; "tenso-relaxado": +1; "áspero-liso": +1 ; "feio-bonito": +1; "pesado-leve": +1; "negativo-positivo": +1; "duro-macio": 0; "maubom": +1; "desagradável-agradável": +3. Os valores das medianas das avaliações dos estímulos D1 feitas pelo Grupo Controle foram os seguintes: "triste-alegre": +1,5; "tenso-relaxado": +2; "áspero-liso": 0; "feio-bonito": 0; "pesado-leve": 0 ; "negativo-positivo": +2; "duro-macio": 0 ; "mau-bom": +1; "desagradável-agradável": +1,5. Portanto, as medianas dos valores das avaliações dos estímulos Sr1 e D1 feitas pelos dois grupos situaram-se entre 0 e +3 , havendo sobreposição de valores apenas nas escalas "positivo-negativo" e "duro-macio".

As escalas ao centro mostram as avaliações dos estímulos da Classe Triste. As medianas das avaliações do estímulo $\mathrm{Sr} 2$ pelo Grupo Experimental em cada uma das nove escalas do Fator 1 foram as seguintes: "tristealegre": 2,5; "tenso-relaxado": 0,5; "áspero-liso": 0; "feio-bonito": 0 ; "pesado-leve": $-0,5$; "negativo-positivo": 1,5; "duro-macio": 0 ; "maubom": -0,5; “desagradável-agradável”: $-0,5$. Os valores das medianas das avaliações dos estímulos D2 feitas pelo Grupo Controle foram os seguintes: "triste-alegre": -3; "tenso-relaxado": -3; “ásperoliso": -2; "feio-bonito": -2; "pesado-leve": -2; "negativo-positivo": -2,5; "duro-macio": 0; "maubom": -2; “desagradável-agradável": -2. As medianas dos valores das avaliações dos estímulos Sr2 e D2 mostraram uma aparente dissociação dado que os valores obtidos a partir das avaliações dos dois grupos ficaram alocados entre - 0,5 e +2.5 no que se refere à avaliação do $\mathrm{Sr} 2$ e entre 0 e -3 no que se refere à avaliação das faces C2. Houve sobreposição de valores apenas na escala "duromacio". 


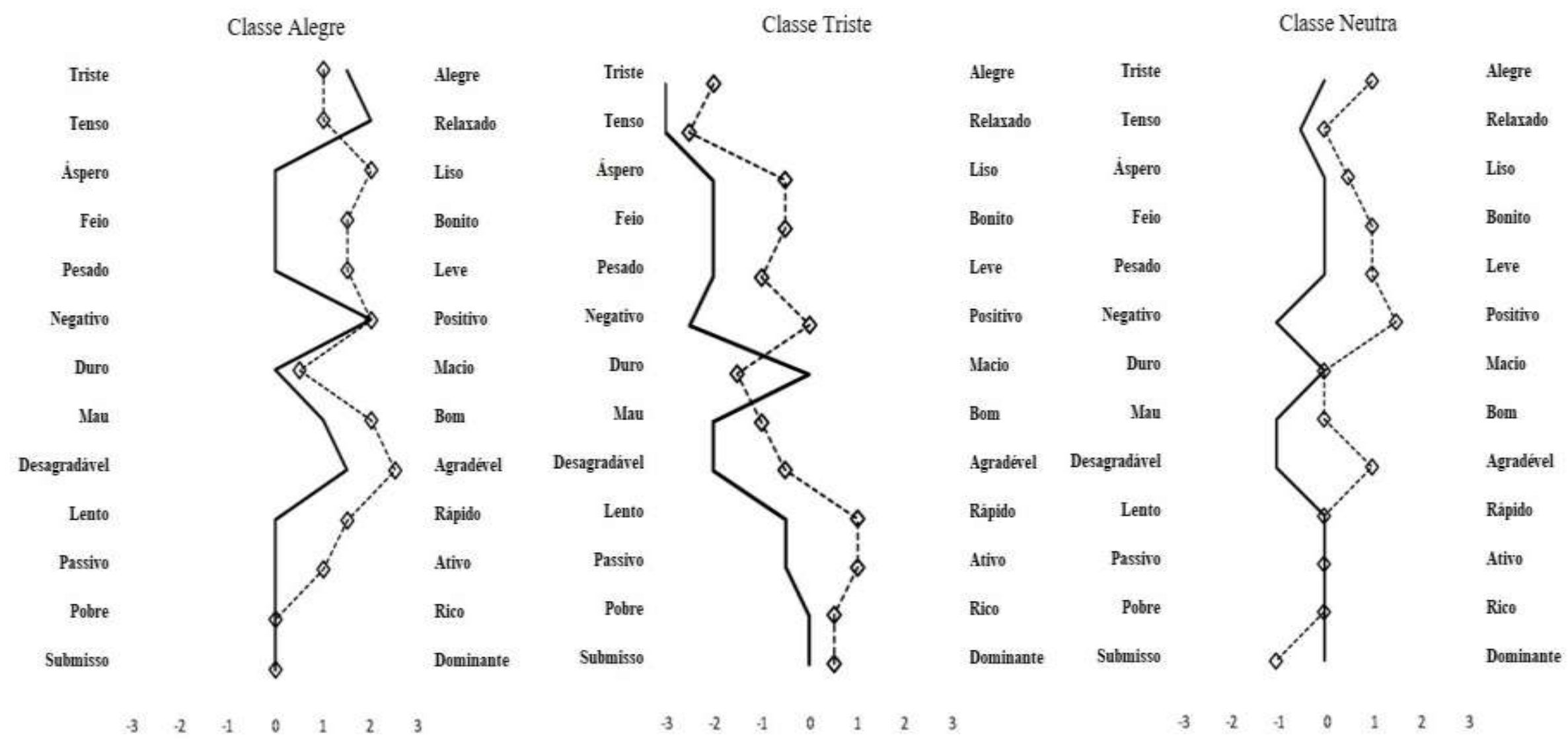

Figura 2. Mediana das avaliações dos estímulos C pelo Grupo Experimental (linhas pontilhadas e losangos) e dos estímulos D pelo Grupo Controle (linhas pretas). 


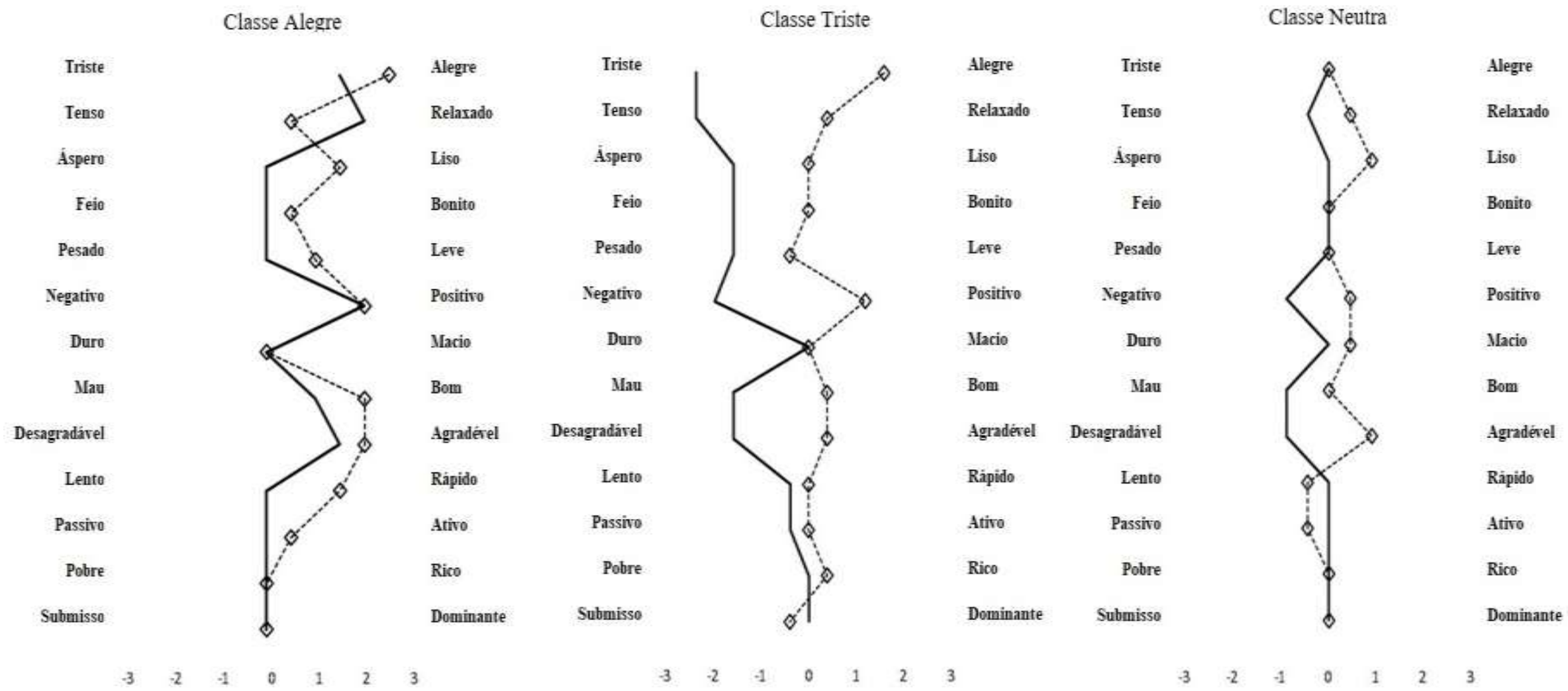

Figura 3. Mediana das avaliações de estímulos Srs pelo Grupo Experimental (linhas pontilhadas e losangos) e dos estímulos D pelo Grupo Controle (linhas pretas). 
As escalas à direita mostram as avaliações dos estímulos da Classe Neutra. As medianas das avaliações do estímulo $\mathrm{Sr} 3$ pelo Grupo Experimental em cada uma das nove escalas do Fator 1 foram as seguintes: "triste-alegre": $+0,5$; "tenso-relaxado": +1,5; "áspero-liso": +1 ; "feiobonito": +1; "pesado-leve": 0; "negativopositivo": +2; "duro-macio": 0; "mau-bom": +1; "desagradável-agradável": +1 . Os valores das medianas das avaliações dos estímulos D3 feitas pelo Grupo Controle foram os seguintes: "tristealegre": 0; "tenso-relaxado": -0,5; "áspero-liso": 0; "feio-bonito": 0; "pesado-leve": 0; "negativopositivo": -1; "duro-macio": 0; "mau-bom": -1; "desagradável-agradável": -1 . Portanto, as medianas dos valores das avaliações dos estímulos Sr3 e D3 feitas pelos dois grupos situaram-se entre -1 e +2 . Houve sobreposição de valores nas escalas "feio-bonito" e "pesado-leve".

\section{DISCUSSÃO}

O objetivo desse experimento foi verificar a emergência de relações arbitrárias e a transferência de função de faces expressando emoções para estímulos abstratos após um treino a partir do procedimento MTS de identidade com consequências específicas. Seis universitários aprenderam a relacionar condicionalmente estímulos antecedentes tendo como base a similaridade física entre eles. Cada resposta correta emitida em uma tentativa apresentava estímulos auditivos distintos e, em seguida, os participantes emitiam uma resposta diferencial a um de três logotipos de lojas situadas no campus para produzir pontos. $\mathrm{O}$ encadeamento de respostas com funções diferentes contrasta com a programação usual de tentativas de MTS, em que a consequência diferencial para acertos é apresentada imediatamente após a escolha do estímulo de comparação experimentalmente definido como correto (cf. Arntzen, 2012; Silveira $\&$ de Rose, 2015). No Experimento 1 do presente estudo, a resposta de consumação às consequências específicas parece ter sido eficaz para que os participantes apresentassem desempenhos consistentes de formação de classes após aprenderem uma série de relações de identidade. Estes resultados replicaram e estenderam os achados de estudos anteriores com indivíduos com desenvolvimento atípico (Dube et al., 1987; Dube et al., 1989; Dube \& McIlvane, 1995) e crianças com desenvolvimento típico (Goyos, 2000; Schenk, 1994). Além disso, os resultados do Experimento 1 parecem ter confirmado a efetividade da resposta de consumação em contingências experimentais (cf. Costa et al., 2007; Galizio \& Buskist, 1988; Kangas \& Hackenberg, 2007; Matthews, Schimoff, Catania, \& Sagvolden 1977).

\section{Formação de classes de equivalência}

P6 exibiu prontamente a emergência das relações arbitrárias entre os estímulos antecedentes $\mathrm{AB}$ (A1B1, A2B2 e A3B3) e BA (B1A1, B2A2 e $\mathrm{B} 3 \mathrm{~A} 3$ ), e entre as combinações de logotipos com estímulos auditivos apresentados como modelos e os estímulos As e Bs como estímulos de comparação: SrA (Sr1A1, Sr2A2 e Sr3A3) e SrB (Sr1B1, Sr2B2 e Sr3B3). Os demais participantes foram submetidos novamente ao bloco de Linha de Base Cumulativa 1. Em seguida, eles apresentaram a emergência de todas estas relações arbitrárias. Estes resultados podem ser interpretados como evidências de emergência atrasada, na medida em que a experiência prévia com relações arbitrárias no procedimento MTS parece influenciar os desempenhos nas tentativas que testam relações de equivalência (cf. Dube et al., 1987, 1989; Dube \& McIlvane, 1995; Haimson, Wilkinson, Rosenquist, Ouimet, \& McIlvane, 2009).

Nos blocos de teste subsequentes ao treino $\mathrm{CC}$ os participantes apresentaram prontamente a emergência das relações $\mathrm{AC}$ (A1C1, A2C2 e $\mathrm{A} 3 \mathrm{C} 3), \mathrm{CA}$ (C1A1, C2A2 e C3A3), BC (B1C1, B2C2 e B3C3), CB (C1B1, C2B2, C3B3) e SrC (Sr1C1, Sr2C2 e Sr3C3). Portanto, o treino de relações de identidade a partir do procedimento MTS com consequências específicas estabeleceu condições para que três classes de equivalência, originalmente com três membros cada, se expandissem para incluir os estímulos Cs: A1B1C1Sr1, A2B2C2Sr2 e A3B3C3Sr3.

\section{Transferência de funções}

As medianas das avaliações observadas nas escalas dos diferenciais semânticos mostraram que os estímulos abstratos e as faces expressando emoções receberam classificações que estavam de acordo com as classes de equivalência formadas a partir do treino. Isto é, o estímulo $\mathrm{C} 1$, relacionado a D1 via $\mathrm{Sr} 1$, recebeu avaliações positivas; o estímulo $\mathrm{C} 2$, relacionado a $\mathrm{D} 2$ via $\mathrm{Sr} 2$, recebeu avaliações negativas, e o estímulo $\mathrm{C} 3$, relacionado a D3 via $\mathrm{Sr} 3$, recebeu avaliações neutras. Os estímulos Cs e Ds compartilhavam apenas relações comuns com os mesmos reforçadores específicos. Eles jamais foram apresentados conjuntamente, como estímulos-modelo e comparações, em tentativas de teste. Por isso, as avaliações dos estímulos Cs pelo Grupo Experimental sugerem que as Srs atuaram como nódulos para a transferência de funções emocionais dos estímulos Ds. As avaliações dos estímulos Cs pelos participantes do Grupo Experimental podem ser consideradas como evidências adicionais da ocorrência de expansão das classes ABCSr para a inclusão dos estímulos D. Portanto, os treinos em MTS de identidade com consequências específicas teriam estabelecido três classes de estímulos com 
cinco membros: A1B1C1D1Sr1, A2B2C2D2Sr2 e A3B3C3D3Sr3.

Resultados robustos de transferência de funções emocionais positivas e negativas para estímulos abstratos também foram observados nos estudos de Almeida e de Rose (2015), Bortoloti e de Rose (2005, 2007, 2009) e Silveira e de Rose (2015). Estes resultados estendem os achados de estudos prévios (cf. Barros et al., 2006; Dube et al., 1987, 1989; Dube \& McIlvane, 1995; Goyos, 2000; Joseph et al., 1998; Johnson et al., 2014; Minster et al., 2006; Monteiro \& Barros, 2016; Schenk, 1994; Varella \& de Souza, 2014, 2015) por demonstrar a possiblidade de que desempenhos que caracterizam a transferência de funções podem ocorrer a partir de reforçadores como nódulos.

Uma novidade apresentada por esta pesquisa teve relação com o requerimento para que os participantes avaliassem os logotipos das lojas por meio do diferencial semântico, após a condução dos procedimentos que formavam classes de estímulos entre estímulos abstratos, faces expressando emoções e consequências específicas. Buscou-se verificar se, após atuarem como nódulos para a formação das classes e para a transferência das funções, todos os logotipos seriam avaliados de maneira similar à avaliação dos demais estímulos. Os resultados obtidos no Experimento 1 podem ser considerados como uma confirmação parcial a esta hipótese, pois se verificou que apenas dois dos três logotipos receberam avaliações similares às das faces: $\mathrm{Sr} 1 \mathrm{e}$ Sr3. Estes resultados chamaram a atenção porque as avaliações dos estímulos C1, C2 e C3 foram semelhantes às avaliações dos estímulos D1, D2 e D3, indicando que os estímulos Sr1, Sr2 e Sr3 participavam das classes de equivalência.

\section{EXPERIMENTO 2}

Os participantes do Experimento 1 apresentaram desempenhos consistentes com a formação de equivalência e transferência de função. E, mais do que demonstrar que as consequências específicas podem atuar como nódulos para ambos os processos, os resultados confirmam a efetividade dos procedimentos que foram desenvolvidos para apresentar as consequências específicas nas tentativas de treino das discriminações condicionais de linhas de base.

O Experimento 2 buscou avaliar a efetividade do procedimento de resposta de consumação empregando-o em tentativas de reversão de consequências específicas que visam a reorganização das classes de equivalência (cf. Dube et al. 1987, 1989; Dube \& McIlvane, 1995) e a reversão da transferência das funções (cf. Almeida \& de Rose, 2015).

\begin{abstract}
MÉTODO
Participantes, Materiais, Estímulos

Para o Experimento 2 foram empregados os mesmos materiais e estímulos que foram utilizados para o Experimento 1. Todos os participantes do Experimento 1 foram submetidos aos procedimentos do Experimento 2, que duravam aproximadamente 25 minutos.
\end{abstract}

\section{Procedimento}

Reversão de Consequências Específicas.

Nesse experimento o responder o responder aos estímulos $\mathrm{C} 1$ e D1 produziam $\mathrm{Sr} 2$ e o responder aos estímulos C2 e D2 produziam o Sr1. Houve, portanto, a reversão entre os dois estímulos empregados como consequências - Sr1 e Sr2 - para o responder às relações entre estímulos das Classes 1 e 2 (C1C1-Sr2, D1D1-Sr2; C2C2-Sr1 e D2D2$\mathrm{Sr} 1)$. As demais características do treino foram idências às do treino utilizado no Experimento 1. Visando verificar se o procedimento de reversão de Srs causaria uma deterioração em todo o desempenho dos participantes, as relações entre $\mathrm{Sr} 3$ e os estímulos da Classe 3 permaneceram inalteradas. Por isso, as respostas aos estímulos C3 e D3, bem como a todos os demais estímulos da Classe 3, produziam consistentemente $\mathrm{Sr} 3$.

No primeiro bloco de reversão, cada discriminação condicional CC e DD foi apresentadas oito vezes, totalizando 24 tentativas de cada relação. O bloco de treino subsequente, Linha de Base Cumulativa 4, continha todas as discriminações condicionais AA, BB, CC e DD. O critério para avançar nestes blocos de treino era 93\% de acertos (cf. Arntzen, 2012). Em seguida, os participantes eram submetidos aos blocos de teste $\mathrm{AB}, \mathrm{BA}, \mathrm{AC}, \mathrm{CA}, \mathrm{BC}, \mathrm{CB}, \mathrm{SrA}, \mathrm{SrB}$ e SrC, com o objetivo de verificar se os participantes responderiam sob controle de novas relações $\mathrm{AC}$ (A1C2 e A2C1), CA (C2A1 e C1A2, BC (B1C2 e B2C1), CB (C1B2 e C2B1) e SrC (Sr1C2 e Sr2C1) - testes de reorganização - e para verificar a manutenção das relações originais $\mathrm{AB}, \mathrm{BA}, \mathrm{SrA}$, $\mathrm{SrB}, \mathrm{A} 3 \mathrm{~B} 3, \mathrm{~B} 3 \mathrm{~A} 3, \mathrm{~A} 3 \mathrm{C} 3, \mathrm{C} 3 \mathrm{~A} 3$ e Sr3C3 - testes de manutenção. Os participantes que obtivessem pelo menos $90 \%$ de acerto nos blocos de teste que envolviam os estímulos C (AC, CA, BC e CB e $\mathrm{SrC}$ ), foram conduzidos a uma nova fase para avaliar os estímulos Cs e Srs por meio do diferencial semântico.

\section{Aplicação do Diferencial Semântico \\ Grupo Experimental. Os participantes que} atingiram os critérios foram conduzidos a uma sala separada na qual puderam avaliar os estímulos $\mathrm{C} 1$, C2, C3, Sr1, Sr2 e Sr3 por meio do diferencial semântico.

Grupo Controle. Os resultados dos participantes que integraram o Grupo Controle do Experimento 1 foram utilizados no Experimento 2. 


\section{RESULTADOS}

\section{Reorganização das classes de equivalência}

A Tabela 2 mostra os desempenhos dos participantes nos blocos de treino e teste. Todos os participantes atingiram os critérios para avançar os blocos na condição de treino. Para cinco dos seis participantes, o responder foi controlado pelas novas relações emergentes estabelecidas graças às reversões dos reforçadores específicos realizadas no presente experimento. Para P1, P2, P3 e P5, a reversão de reforçadores estabeleceu contexto para que os membros das classes de equivalência se reorganizassem para formar as novas classes $\mathrm{A} 1 \mathrm{~B} 1 \mathrm{C} 2 \mathrm{Sr} 2$ e A2B2C1Sr1. O participante P4 não atingiu os critérios para atestar a reorganização de classes. As porcentagens de acertos baixas nos testes de reorganização e as concomitantes altas porcentagens de acertos nos testes de manutenção parecem indicar que, para $\mathrm{P} 4$, houve manutenção das relações de equivalência que haviam sido estabelecidas pelas contingências de treino do Experimento 1.

Tabela 2

Porcentagens de Acertos dos Participantes nos Blocos de Treino e Teste do Experimento 2

\begin{tabular}{|c|c|c|c|c|c|c|c|}
\hline \multirow[b]{2}{*}{ Fases } & \multirow[b]{2}{*}{ Critério } & \multicolumn{6}{|c|}{ Participantes } \\
\hline & & P1 & $\mathrm{P} 2$ & P3 & P4 & P5 & P6 \\
\hline Treino CC e DD & 92 & 100 & 100 & 100 & 100 & 100 & 100 \\
\hline $\begin{array}{l}\text { Linha de Base } \\
\text { Cumulativa } 4\end{array}$ & 100 & 100 & 100 & 100 & 100 & 100 & 100 \\
\hline $\begin{array}{l}\text { Teste de Manutenção } \\
\text { AB }\end{array}$ & 90 & 86 & 100 & 100 & 100 & 100 & 100 \\
\hline $\begin{array}{c}\text { Teste de Manutenção } \\
\text { BA }\end{array}$ & 90 & 100 & 100 & 100 & 100 & 100 & 100 \\
\hline $\begin{array}{c}\text { Teste de } \\
\text { Reorganização AC }\end{array}$ & 90 & 93 & 100 & 100 & 40 & 100 & 100 \\
\hline $\begin{array}{c}\text { Teste de } \\
\text { Reorganização CA }\end{array}$ & 90 & 100 & 100 & 93 & 40 & 100 & 100 \\
\hline $\begin{array}{c}\text { Teste de } \\
\text { Reorganização BC }\end{array}$ & 90 & 100 & 100 & 100 & 33 & 100 & 100 \\
\hline $\begin{array}{c}\text { Teste de } \\
\text { Reorganização CB }\end{array}$ & 90 & 100 & 100 & 100 & 33 & 100 & 100 \\
\hline $\begin{array}{l}\text { Teste de Manutenção } \\
\text { SrA }\end{array}$ & 90 & 100 & 100 & 100 & 100 & 100 & 100 \\
\hline $\begin{array}{l}\text { Teste de Manutenção } \\
\text { SrB }\end{array}$ & 90 & 100 & 100 & 100 & 100 & 100 & 100 \\
\hline $\begin{array}{c}\text { Teste de } \\
\text { Reorganização SrC }\end{array}$ & 90 & 100 & 100 & 100 & 13 & 93 & 100 \\
\hline
\end{tabular}

\section{Análise dos diferenciais semânticos}

A Figura 4 mostra as medianas dos valores das avaliações feitas por cinco participantes do Grupo Experimental dos estímulos Cs (linhas tracejadas) e as medianas das avaliações feitas pelo Grupo Controle dos estímulos D1, D2 e D3 (linhas cheias). Para facilitar a apresentação dos resultados, os estímulos C1 e Sr1 serão chamados de "Classe Alegre-Triste", os estímulos C2 e Sr2 serão chamados de "Classe Triste-Alegre", porque esperava-se que as reversões D1-Sr2 e D2-Sr1 ocasionassem a reversão da transferência das funções emotivas para os estímulos abstratos $\mathrm{C} 1$ e C2. Os estímulos C3 e Sr3 continuaram sendo "Classe Neutra", pois não houve manipulação da relação entre esses estímulos. O mesmo raciocínio foi empregado para nomear as escalas que apresentam os resultados das avaliações de Sr1, Sr2 e $\mathrm{Sr} 3$.

As escalas à esquerda da Figura 4 mostram as avaliações feitas dos estímulos da Classe Alegre-
Triste. As medianas das avaliações do estímulo $\mathrm{C} 1$ pelo Grupo Experimental em cada uma das nove escalas do Fator 1 foram as seguintes: "tristealegre": -0,5; "tenso-relaxado": -1; "áspero-liso": 1; "feio-bonito": 0; "pesado-leve": -0,5; "negativopositivo": 0; "duro-macio": -1; "mau-bom": 0 ; "desagradável-agradável": -0,5. Os valores das medianas das avaliações dos estímulos D2 feitas pelo Grupo Controle foram os seguintes: "tristealegre": -3; "tenso-relaxado": -3; "áspero-liso": -2; "feio-bonito": -2; "pesado-leve": -2; "negativopositivo": -2,5; "duro-macio": 0; "mau-bom": -2; "desagradável-agradável": $\quad-2$. Portanto, as medianas dos valores das avaliações dos estímulos C1 e D2 feitas pelos dois grupos situaram-se entre 0 e -3. Não houve sobreposição de valores em nenhuma das escalas. Estes resultados mostram que ambos os estímulos possuíam valências afetivas negativas.

As escalas ao centro mostram as avaliações feitas dos estímulos da Classe Triste- 
Alegre. As medianas das avaliações do estímulo C2 pelo Grupo Experimental em cada uma das nove escalas do Fator 1 foram as seguintes: "tristealegre": 2; "tenso-relaxado": 0; "áspero-liso": -0.5; "feio-bonito": 0,5; "pesado-leve": -0,5; "negativopositivo": 0; "duro-macio": -1; "mau-bom": 0 ; "desagradável-agradável": +1. Os valores das medianas das avaliações dos estímulos D1 feitas pelo Grupo Controle foram os seguintes: "tristealegre": +1,5; "tenso-relaxado": +2; "áspero-liso": 0; "feio-bonito": 0; "pesado-leve": 0; "negativopositivo": +2; "duro-macio": 0 ; "mau-bom": +1 ; "desagradável-agradável": +1,5. Portanto, as medianas dos valores das avaliações dos estímulos C2 feitas pelo Grupo Experimental apresentaram valores positivos em três escalas ("triste-alegre", "feio-bonito"; "desagradável-agradável"). Por outro lado, as avaliações dos estímulos D1 feitas pelo Grupo Controle foram positivas em praticamente todas as escalas (entre 0 e +2 ). Não houve sobreposição de valores em nenhuma das escalas do Fator 1. Os resultados mostram que as valências afetivas dos estímulos foram análogas em apenas algumas escalas.

As escalas à direita mostram as avaliações dos estímulos da Classe Neutra. As medianas das avaliações do estímulo C3 pelo Grupo Experimental em cada uma das nove escalas do Fator 1 foram as seguintes: "triste-alegre": 0; "tenso-relaxado": +0,5; "áspero-liso": +1 ; "feiobonito": 0; "pesado-leve": 0; "negativo-positivo": $+0,5$; "duro-macio": +0 ; "mau-bom": 0 ; "desagradável-agradável": +1 . Os valores das medianas das avaliações dos estímulos D3 feitas pelo Grupo Controle foram os seguintes: "tristealegre": 0; "tenso-relaxado": -0,5; "áspero-liso": 0; "feio-bonito": 0; "pesado-leve": 0; "negativopositivo": -1; "duro-macio": 0; "mau-bom": -1; "desagradável-agradável": $\quad-1$. Portanto, as medianas dos valores das avaliações dos estímulos C3 e D3 feitas pelos dois grupos situaram-se entre 1 e +1 . Houve sobreposição de valores apenas nas escalas "feio-bonito" e "pesado-leve". Estes resultados mostram que ambos os estímulos possuíam valências neutras.

As escalas à esquerda da Figura 5 mostram as avaliações dos estímulos da Classe Alegre-Triste. As medianas das avaliações do estímulo Sr1 pelo Grupo Experimental em cada uma das nove escalas do Fator 1 foram as seguintes: "triste-alegre": $+1,5$; "tenso-relaxado": 0; "áspero-liso": +1; "feiobonito": +0,5; "pesado-leve": $+0,5$; "negativopositivo": +0,5; "duro-macio": $+0,5$; "mau-bom": $+0,5$; "desagradável-agradável": +1 . Os valores das medianas das avaliações dos estímulos D2 feitas pelo Grupo Controle foram os seguintes: "tristealegre": -3; "tenso-relaxado": -3; "áspero-liso": -2; "feio-bonito": -2; "pesado-leve": -2; "negativopositivo": -2,5; "duro-macio": 0; "mau-bom": -2; "desagradável-agradável”: $\quad-2$. Os resultados mostram que as medianas dos valores das avaliações do estímulo Sr1 ficaram entre +0,5 e $+1,5 \mathrm{em}$ praticamente todas as escalas do Fator 1 . Houve uma redução na positividade das avaliações em relação aos resultados do Experimento 1. Porém, as avaliações deste estímulo não foram condizentes com as avaliações de D2, feitas pelo Grupo Controle que se situaram entre 0 e -3 . Os valores das medianas se sobrepuseram em uma única escala: "duro-macio".

As escalas ao centro mostram as avaliações feitas dos estímulos da Classe TristeAlegre. As medianas das avaliações do estímulo Sr2 pelo Grupo Experimental em cada uma das nove escalas do Fator 1 foram as seguintes: "tristealegre": +0,5; "tenso-relaxado": 0; "áspero-liso": +0.5 ; "feio-bonito": +0,5; "pesado-leve": 0 ; "negativo-positivo": - 0,5 ; "duro-macio": +0,5; "mau-bom": -0,5; “desagradável-agradável": 0. Os valores das medianas das avaliações dos estímulos D1 feitas pelo Grupo Controle foram os seguintes: "triste-alegre": +1,5; "tenso-relaxado": +2; "áspero-liso": 0; "feio-bonito": 0; "pesado-leve": 0; "negativo-positivo": +2; "duro-macio": 0 ; "maubom": +1; "desagradável-agradável": +1,5. Portanto, as medianas dos valores das avaliações dos estímulos $\mathrm{Sr} 2$ feitas pelo Grupo Experimental apresentaram valores positivos em quatro escalas ("triste-alegre", “áspero-liso"; “feio-bonito"; “desagradável-agradável"). Por outro lado, as avaliações dos estímulos D1 feitas pelo Grupo Controle foram positivas em praticamente todas as escalas (entre 0 e +2 ). Não houve sobreposição de valores em nenhuma das escalas do Fator 1. Os resultados mostram uma relativa modificação da valência afetiva do estímulo $\mathrm{Sr}$, que passou a ser avaliado como mais positivo, quando comparado às avaliações dos estímulos D1.

As escalas à direita mostram as avaliações feitas dos estímulos da Classe Neutra. As medianas das avaliações do estímulo C3 pelo Grupo Experimental em cada uma das nove escalas do Fator 1 foram as seguintes: "triste-alegre": +1; "tenso-relaxado": 0 ; "áspero-liso": +1 ; "feiobonito": +0,5; "pesado-leve": +1 ; "negativopositivo": +0,5; "duro-macio": 0; "mau-bom": $+0,5$; “desagradável-agradável": 0 . Os valores das medianas das avaliações dos estímulos D3 feitas pelo Grupo Controle foram os seguintes: "tristealegre": 0; "tenso-relaxado": -0,5; "áspero-liso": 0; "feio-bonito": 0; "pesado-leve": 0; "negativopositivo": -1; "duro-macio": 0; "mau-bom": -1; "desagradável-agradável": $\quad-1$. Portanto, as medianas dos valores das avaliações dos estímulos C3 e D3 feitas pelos dois grupos situaram-se entre 1 e +1 . Houve sobreposição de valores apenas nas escalas "feio-bonito" e "pesado-leve". Estes resultados mostram que ambos os estímulos possuíam valências neutras. 


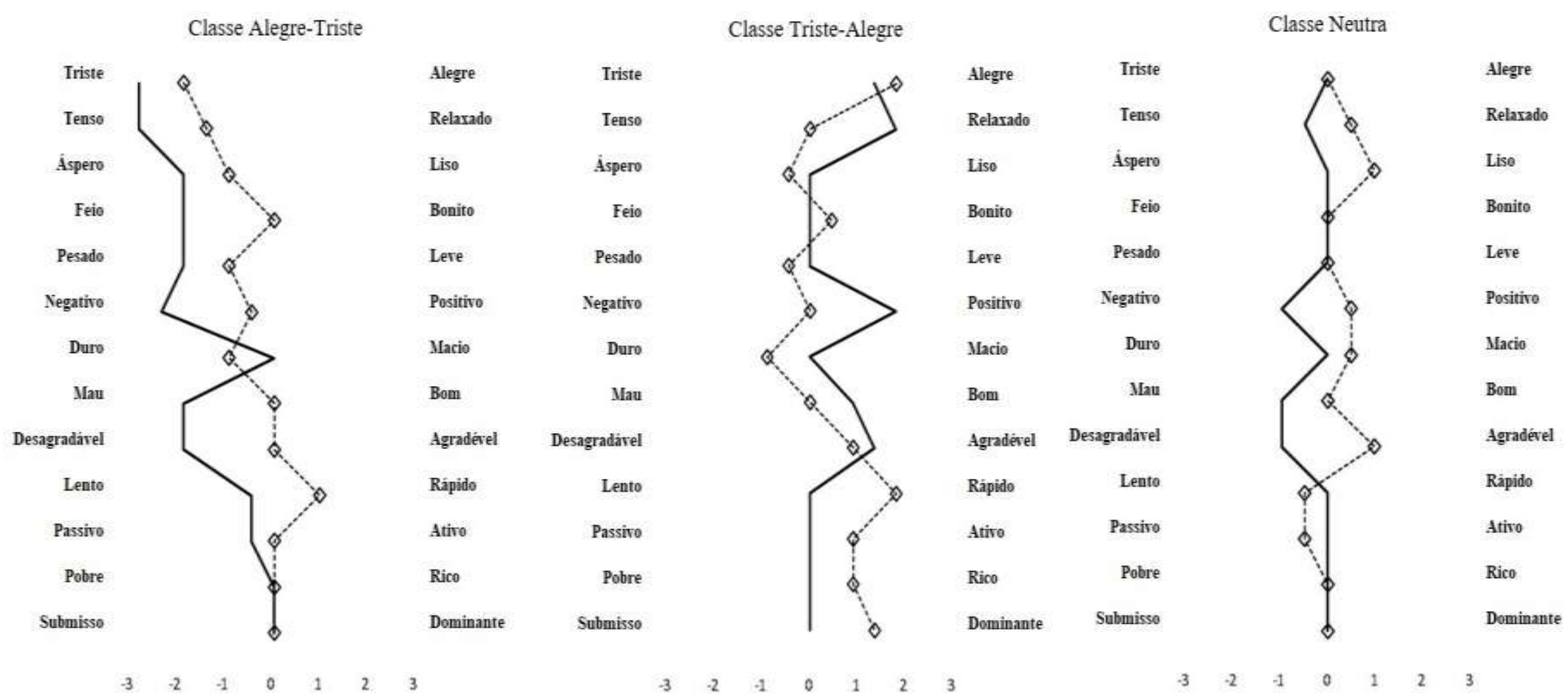

Figura 4. Mediana das avaliações dos estímulos C pelo Grupo Experimental (linhas pontilhadas e losangos) e dos estímulos D pelo Grupo Controle (linhas pretas). 


\section{DISCUSSÃO}

\section{Reorganização das classes de equivalência \\ Cinco dos seis participantes}

demonstraram a reorganização das relações de equivalência após a reversão de reforçadores específicos para duas classes de estímulos. Estes participantes responderam sob controle das relações $\mathrm{AB}, \mathrm{BA}, \mathrm{A} 3 \mathrm{C} 3, \mathrm{C} 3 \mathrm{~A} 3, \mathrm{~B} 3 \mathrm{C} 3, \mathrm{C} 3 \mathrm{~B} 3$, $\mathrm{SrA}, \mathrm{SrB}$ e $\mathrm{Sr} 3 \mathrm{C} 3$ estabelecidas previamente e sob controle de novas relações $\mathrm{A} 1 \mathrm{C} 2, \mathrm{~A} 2 \mathrm{C} 1, \mathrm{C} 1 \mathrm{~A} 2$, A2C1, B1C2, B2C1, C1B2, C2B1, Sr1C2 e Sr2C1. Estes desempenhos sugerem a reorganização dos membros nas classes de estímulos originais que formaram duas novas classes: A1B1C2Sr2 e A2B2C1Sr1. Um participante apresentou a manutenção das relações estabelecidas no Experimento 1. Contudo, nas tentativas que testavam a reorganização das classes, os estímulos de comparação aparentemente exerceram um controle misto sobre as respostas de escolha. Tais desempenhos parecem indicar que o procedimento de reversão de reforçadores resultou na fusão dessas classes (cf. Sidman, 1994, 2000).

O Experimento 2 replicou e estendeu os achados de reorganização de classes a partir de treinos em MTS com reforçadores específicos para participantes humanos com desenvolvimento típico. Os resultados de cinco participantes fortalecem os resultados positivos acerca da eficácia da utilização de respostas de consumação em procedimentos MTS com reforçadores específicos tanto para o estabelecimento quanto para a reversão dos desempenhos de linha de base.

\section{Reversão da transferência de função}

As escalas do diferencial semântico mostraram que os valores das medianas das avaliações dos estímulos D1 e D2 foram revertidos. Ou seja, o estímulo D1, originalmente avaliado como positivo, foi avaliado como negativo e o estímulo D2, orginalmente avaliado como negativo, foi avaliado como positivo. Estas reversões nos resultados da transferência de função parecem ter ocorrido em função do procedimento de reversão de reforçadores que oportunizou a realocação dos estímulos D1 e D2 nas classes de equivalência reorganizadas: A1B1C2D2Sr2 e A2B2C1D1Sr1. Além de confirmar a inclusão dos estímulos D1 e D2 nas classes reorganizadas, os resultados obtidos a partir dos diferenciais semânticos estendem os resultados do Experimento 1 na medida em que demonstram que as funções dos estímulos abstratos, estabelecidas por meio de processos de transferência de função, dependem das contingências de treino.

O procedimento de treino utilizado no Experimento 2 para reverter a transferência de função (classes Triste-Alegre e Alegre-Triste) diferiu do procedimento de Almeida e de Rose (2015), que explicitamente alterou as relações de controle entre estímulos modelo e estímulos de comparação em um procedimento MTS arbitrário com uma consequência comum. No presente experimento, desempenhos análogos foram obtidos após a reversão das relações entre estímulos antecedentes e estímulos reforçadores específicos em um procedimento de MTS de identidade. Tomados em conjunto, estes dados corroboram a noção de que as diversas modalidades de responder relacional derivado seriam produtos das contingências de reforço (Sidman, 1994, 2000). Futuros estudos deverão investir na verificação dos efeitos de reversão de reforçadores sobre a transferência de função, no contexto de MTS de identidade, de todas as classes de equivalência ao invés de apenas duas, como na presente pesquisa.

\section{DISCUSSÃO GERAL}

O principal objetivo da presente pesquisa foi determinar se após o treino em um procedimento MTS de identidade com reforçadores específicos, em que havia a exigência de uma resposta de consumação a cada um dos reforçadores durante as tentativas de MTS, humanos adultos passariam a responder sob controle de relações arbitrárias entre estímulos antecedentes. Além disso, indagou-se sobre a possibilidade de ser verificada a transferência de função de faces humanas para os conjuntos de estímulos abstratos, uma vez que os estímulos reforçadores passaram a ser comuns a estes elementos. Ambos os experimentos foram bem-sucedidos no que se refere a estes objetivos, especialmente se contrastados com o insucesso em se observar tais fenômenos reportados anteriormente (Silveira \& de Rose, 2015).

Aproveitamos o ensejo para destacar a novidade deste estudo que foi demonstrar resultados que sugerem a ocorrência transferência de função mensurados após a formação e a reorganização de classes de equivalência estabelecidas a partir de um procedimento MTS de identidade com consequências específicas. As avaliações dos estímulos abstratos e por meio do diferencial semântico mostraram que a transferência de função é mais robusta quando estes estímulos integram a mesma classe que as faces expressando alegria. Resultados análogos foram reportados em praticamente todos os estudos conduzidos anteriormente (cf. Aggio et al., 2014; Almeida \& de Rose, 2015; Bortoloti \& de Rose, 2011, Bortoloti et al., 2013; Silveira et al. 2016; Silveira, Mackay, \& de Rose, 2017). 
Classe Alegre-Triste

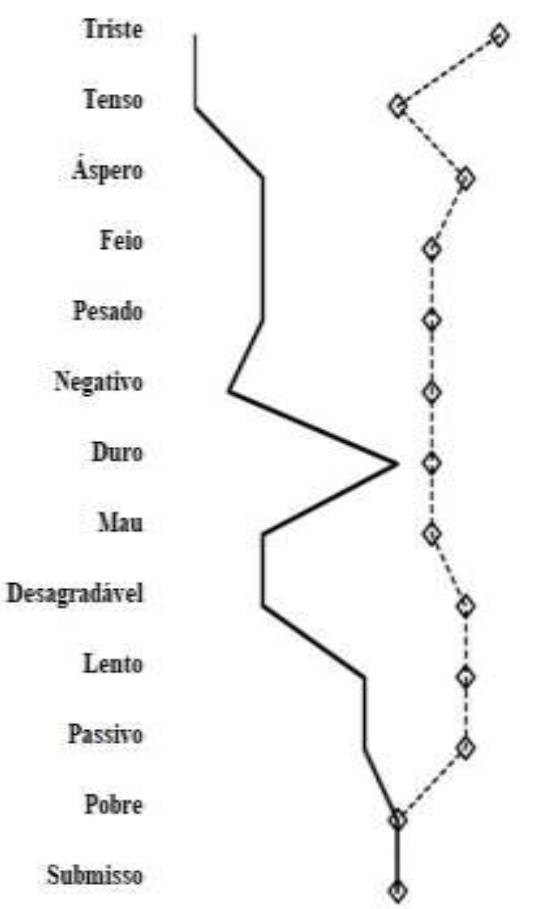

$\begin{array}{lr}\text { Alegre } & \text { Triste } \\ \text { Relasado } & \text { Tenso } \\ \text { Liso } & \text { Aspero } \\ \text { Bonito } & \text { Feio } \\ \text { Leve } & \text { Pesado } \\ \text { Positivo } & \text { Negativo } \\ \text { Macio } & \text { Duro } \\ \text { Bom } & \text { Mau } \\ \text { Agradével } & \text { Desagradivel } \\ \text { Rápido } & \text { Lento } \\ \text { Ativo } & \text { Passivo } \\ \text { Rico } & \text { Pobre } \\ \text { Dominante } & \text { Submisso }\end{array}$

\section{Classe Triste-Alegre}

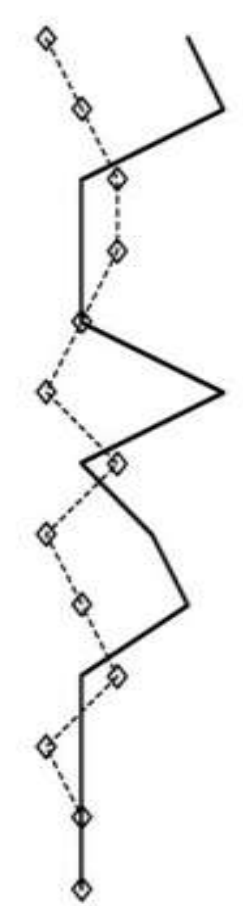

Alegre
Relasado
Liso
Bonito
Leve
Positivo
Macio
Bom
Agradével
Rapido
Ativo
Rico
Dominante

Classe Neutra

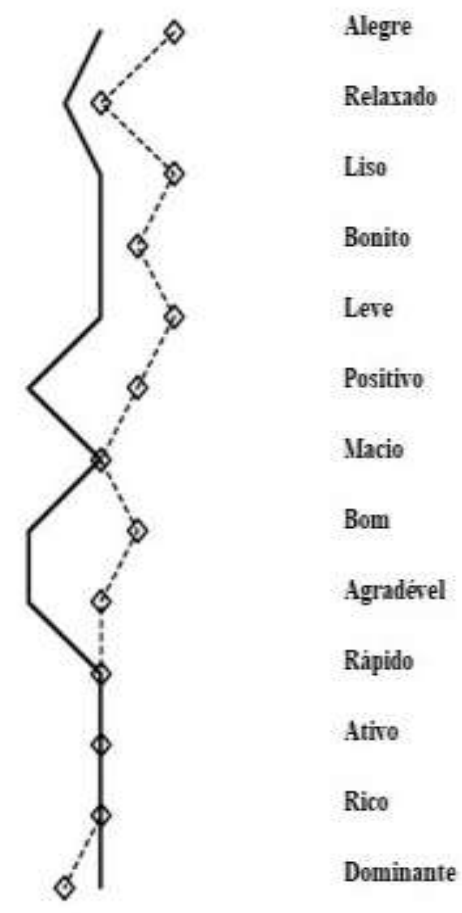

Figura 5. Mediana das avaliações de estímulos Sr pelo Grupo Experimental (linhas pontilhadas e losangos) e dos estímulos D pelo Grupo Controle (linhas pretas). 
Tomados em conjunto, os resultados deste estudo confirmam que estímulos com valência afetiva positiva, como é o caso de um conjunto de faces alegres, influenciam o grau de relacionamento entre os estímulos que compõem uma classe. Mas apesar dos avanços significativos obtidos neste estudo, é importante destacar aqui que a metodologia empregada na presente pesquisa para aferir a transferência de função das faces para os estímulos abstratos apresentou uma falha importante, porque não houve balanceamento adequado no número de participantes no Grupo Controle e no Grupo Experimental. Na ausência de um balanceamento adequado ficou impossível fazer uso de tratamento estatístico que pudesse confirmar que as avaliações dos estímulos D, por exemplo, pelos participantes do Grupo Experimental teriam correlação com as avaliações dos estímulos E pelo Grupo Controle. Estando reféns da comparação por meio de inspeção visual, é possível afirmar que há fortes indícios de ocorrência de transferência de funções. Entretanto, há a necessidade de estudos adicionais com controle mais rigoroso do $\mathrm{N}$ para a realização dos testes estatísticos adequados. Outra alternativa seria utilizar a metodologia de pesquisa característica da análise do comportamento que é o uso de sujeito como seu próprio controle para avaliar a transferência de função a partir de procedimentos MTS com reforçadores específicos.

É importante também considerar os possíveis efeitos de outras variáveis experimentais que poderiam ter relação com os desempenhos descritos. Em primeiro lugar, cada uma das consequências específicas envolvia estímulos complexos que embutiam sons, imagens e acréscimo de pontos. Será que desempenhos de formação, reversão e transferência de funções seriam obtidos se estímulos com menor grau de complexidade fossem empregados como consequências específicas? Em segundo lugar, os participantes atribuiriam valores diferentes para os pontos ganhos para as respostas de consumação para cada logotipo (correspondentes a diferentes tipos de brindes)? Estes reforçadores de diferentes magnitudes por si só afetariam os resultados de formação, reorganização e transferência de função? Considera-se, portanto, importante encorajar estudos futuros que visem elucidar os efeitos de tais variáveis.

\section{REFERÊNCIAS}

Aggio, N. M., Almeida, J. H., Cortez, M. D., \& de Rose, J. C. (2014). O papel das emoções na aprendizagem do comportamento simbólico. Perspectivas em Análise do Comportamento, 5, 27-39.

Almeida, J. H., Bortoloti, R., Ferreira, P. R. S., Schielini, P. W., de Rose, J. C. (2014). Análise da validade e precisão de instrumento de diferencial semântico. Psicologia: Reflexão $e$
Crítica, 27, 272-281. doi: 10.1590/16787153.201427207

Almeida, J. H., \& de Rose, J. C. (2015). Changing the meaningfulness of abstract stimuli by the reorganization of equivalence classes: Effects of delayed matching. The Psychological Record, 65, 451-461. doi: 10.1007/s40732-015-0120

Arntzen, E. (2012). Training and testing parameters in formation of stimulus equivalence: Methodological issues. European Journal of Behavior Analysis, 13, 123-135. doi: 10.1080/15021149.2012.11434412

Barros, R. S., Lionello-DeNolf, K. M., Dube, W. V., \& McIlvane, W. J. (2006). Equivalence class formation via identity matching to sample and simple discrimination with class-specific consequences. Revista Brasileira de Análise do Comportamento, 2, 79-92.

Bortoloti, R., \& de Rose, J. C. (2007). Medida do grau de relacionamento entre estímulos equivalentes. Psicologia: Reflexão e Crítica, 20, 252-258. doi: 10.1590/S010279722007000200011

Bortoloti, R., \& de Rose, J. C. (2009). Assessment of the relatedness of equivalent stimuli through a semantic differential. The Psychological Record, 59, 563-590. doi: 10.1007/BF03395682

Bortoloti, R., \& de Rose, J. C. (2011). An Orwellian account of stimulus equivalence: Are some stimuli more equivalent than others? European Journal of Behavior Analysis, 12, 121-134. doi: 10.1080/15021149.2011.11434359

Bortoloti, R., Rodrigues, N., Cortez, M. D., Pimentel, N. S., \& de Rose, J. C. (2013). Overtraining increases the strength of equivalence relations. Psychology and Neurosciensce, 6, 357-364. doi: 10.3922/j.psns.2013.3.13

Capobianco, D., Teixeira, C., Bela, R. E., Orlando, A. F., de Souza, D. G., \& de Rose, J. C. (2009). LECH-GEIC. Sistema web gerenciador de ensino individualizado por computador. (http://geic.ufscar.br:8080/site/)

Costa, C. E., Patsko, C. H., \& Becker, R. M. (2007). Desempenho em FI com humanos: Efeito da instrução, da resposta de consumação e do tipo de instrução. Interação em Psicologia, 11, 175185. doi: 10.5380/psi.v11i2.11609

Dube, W. V., \& McIlvane, W. J. (1995). Stimulusreinforcer relations and emergent maching-tosample. The Psychological Record, 45, 591612.

Dube, W. V., McIlvane, W. J., Mackay, H. A., \& Stoddard. L. T. (1987). Stimulus class membership established via stimulus-reinforcer relations. Journal of the Experimental Analysis of Behavior, 47, 159-175. doi: 10.1901/jeab.1987.47-159

Dube, W. V., McIlvane, W. J., Maguire, R. W., Mackay, H. A., \& Stoddard, L. T. (1989). 
Stimulus class formation and stimulusreinforcer relations. Journal of the Experimental Analysis of Behavior. 51, 65-76. doi: 10.1901/jeab.1989.51-65

Galizio, M., \& Buskist, W. (1988). Laboratory lore and research practices in the experimental analysis of human behavior: Selecting reinforcers and arranging contingencies. The Behavior Analyst, 11, 65-69.

Goyos, C. (2000). Equivalence class formation via common reinforcers among preschool children. The Psychological Record, 50, 629-654. doi: 10.1007/BF03395375

Haimson, B., Wilkinson, K. M., Rosenquist, C., Ouimet, C., \& McIlvane, W. J. (2009). Electrophysiological correlates of stimulus equivalence processes. Journal of the Experimental Analysis of Behavior, 92, 245256. doi: 10.1901/jeab.2009.92-245

Johnson, C., Meleshkevich, O., \& Dube, W. V. (2014). Merging separately established stimulus classes with outcome-specific reinforcement. Journal of the Experimental Analysis of Behavior, 101, 38-50. doi: 10.1002/jeab.61

Joseph, B., Overmier, J. B., \& Thompson, T. (1997). Food and nonfood-related differential outcomes in equivalence learning by adults with Prader-Willi Syndrome. American Journal on Mental Retardation, 101, 374-386.

Kangas, B. D., \& Hackenberg, T. D. (2009). On reinforcing human behavior in laboratory: A brief review and some recommendations. Experimental Analysis of Human Behavior Bulletin, 27, 21-26.

Matthews, B. A., Shimoff, E., Catania, A. C., \& Sagvolden, T. (1977). Uninstructed human responding: sensitivity to ratio and interval contingencies. Journal of the Experimental Analysis of Behavior, 27 (3), 453-467. doi: 10.1901/jeab.1977.27-453

Minster, S. T, Jones, M., Eliffe, D., \& Muthukumaraswamy, S. D. (2006). Stimulus equivalence: Testing Sidman's (2000) theory. Journal of the Experimental Analysis of Behavior, 85, 371-391. doi: 10.1901/jeab.2006.15-05

Monteiro, P. C. M., \& Barros, R. S. (2016). Emergence of auditory-visual relations via equivalence class formation in children diagnosed with autism. The Psychological Record. Advance online publication. doi: 10.1007/s40732-016-0192-1

Sidman, M. (1994). Equivalence relations and behavior: A research story. Boston-MA: Authors Cooperative.

Sidman, M. (2000). Equivalence relations and the reinforcement contingency. Journal of the Experimental Analysis of Behavior, 74, 127 146. doi: 10.1901/jeab.2000.74-127
Sidman, M., \& Tailby, W. (1982). Conditional discrimination vs. matching-to-sample: An expansion of the testing paradigm. Journal of the Experimental Analysis of Behavior, 37, 261273. doi: 10.1901/jeab.1982.37-5

Silveira, M. V., \& de Rose, J. C. (2015). Consequências específicas arbitrárias em Matching de Identidade são efetivas para o estabelecimento de classes de equivalência? Revista Brasileira de Análise do Comportamento. 61-69. doi: 10.18542/rebac.v11i1.3775

Silveira, M. V., Aggio, N. M., Cortez, M. D., Bortoloti, R., Rico, V. V., \& de Rose, J. C. (2016). Maintenance of equivalence classes and transfer of function: Role of the nature of stimuli. The Psychological Record, 66, 65-74. doi: 10.1007/s40732-015-0152-1

Silveira, M. V., Mackay, H. A., \& de Rose, J. C. (2017). Measuring the "transfer of meaning" through members of equivalence classes merged via a class-specific reinforcement procedure. Learning \& Behavior. Advance online publication. doi: 10.3758/s13420-017-0298-6

Varella, A. A. B., \& de Souza, D. G. (2014). Emergence of auditory-visual relations from a visual-visual baseline with auditory-specific consequences in individuals with autism. Journal of the Experimental Analysis of Behavior, 102, 139-149. doi: 10.1002/jeab.93

Varella, A. A. B., \& de Souza, D. G. (2015). Using class specific compound consequences to teach dictated and printed letter relations to a child with autism. Journal of Applied Behavior Analysis, 48, 1-5. doi: 10.1002/jaba.2

Submetido em: 30/08/2017 Aceito em: 30/01/2018 